\title{
The Rational Use of Mucolytic Drugs in Children: Worsening of the Cough as Adverse Drug Reaction after Carbocysteine in Children
}

\author{
Ioan Magyar ${ }^{1,2 *}$, Mihai Botea ${ }^{2}$, Alina Maghiar ${ }^{2}$, Carmen Pantis ${ }^{3}$, Cristian Sava ${ }^{4}$ and Barbu Cuparencu ${ }^{1}$ \\ ${ }^{1}$ Pharmacology Department, Faculty of Medicine \& Pharmacy, University of Oradea, Romania \\ ${ }^{2}$ Emergency Department, Clinical County Hospital Oradea, University of Oradea, Romania \\ ${ }^{3}$ Anaesthesiology \& Intensive Care Unit, Clinical County Hospital Oradea, University of Oradea, Romania \\ ${ }^{4}$ Department of Paediatrics, Clinical Municipal Hospital Oradea, University of Oradea, Romania
}

\begin{abstract}
Carbocysteine and acetylcysteine are drugs with mucolytic and expectorant properties widely used in respiratory disease in adults but also in children. Acetylcysteine is also used as donor of $\mathrm{SH}$ groups in the drug overdose such as in the acetaminophen poisoning. Chronic cough is a vexing and common problem for children, their care givers, and health care providers. Effective treatment may be difficult, especially if the underlying cause of the cough cannot be determined. On the other hand, asthma, recurrent wheezing, bronchiolitis and both upper and lower respiratory tract disease are very common seen in paediatric emergency department. We have tried to found any relationship between use of drugs which are containing carbocysteine and some symptoms such as persistent cough, recurrent wheezing or bronchospasm. We are considering these symptoms as adverse drug reactions of carbocysteine. Our work was focused on 191 children divided in two groups: group A (who received carbocysteine) and group B (without carbocysteine). Our work shows that in all cases of worsening of cough, this effect was credited on the use of carbocysteine. On the other hand, worsening of cough (as frequency and duration) often causes the appearance of reflex vomiting. In case of the late condition the number of hospital admission is increased. Overall we believe that the use of carbocysteine in children exceeds the therapeutic benefit.
\end{abstract}

Keywords: Carbocysteine; Cough; Bronchospasm; Children; Mucolytic

\section{Introduction}

Carbocysteine and acetylcysteine are drugs acting as mucolytics and expectorants. The therapeutic uses of these drugs are mainly as mucolytics of thick viscous and sticky purulent secretions present in the airways, both in adults and children [1,2]. Acetylcysteine in addition to its use in respiratory pathology is also used as an antidote in cases of acute poisoning (overdose) with acetaminophen. However, it is known that acetylcysteine should be used with caution in patients with bronchial hyperactivity, bronchospasm, asthma or other bronchospastic conditions [2,3]. According to some authors, acetylcysteine is even contraindicated in asthma (inducing bronchospasm) [3]. On the other hand, carbocysteine is one of the most prescribed mucolytic drugs in pediatric respiratory pathology, at least in our study. Chronic cough is a vexing and common problem for children, their care givers, and health care providers. Effective treatment may be difficult, especially if the underlying cause of the cough cannot be determined. Etiologies are varied and include common infectious agents that cause both upper and lower respiratory tract disease, asthma, foreign body aspiration and chronic primary pulmonary disease [4] (Figure 1).

Our research was based on the observations made on children who were brought to our emergency department presenting the main symptom of persistent and prolonged irritating cough. This has been mainly related to use of carbocysteine, because in the last medical history (before prescribing carbocysteine) the cough was not so obvious (by the contrary, even absent) on other mucolytics or expectorants when they was used. In other situations, carbocysteine use was associated with a worsening cough, accompanied by bronchospasm and occurrence of vomiting reflex. We consider all these symptoms (cough emergence or worsening of pre-existing bronchospasm and sometimes wheezing) being adverse effects of carbocysteine [5].

\section{Materials and Methods}

This study was performed within the first 4 months of 2011, taking into account all children receiving carbocysteine and who presented in the emergency department with a new onset or worsening of cough and sometimes with the bronchospasm onset. In the mentioned period 89 children who received carbocysteine were recorded. The control group, was made by 102 children who presented with all the respiratory symptoms (such as cough, runny nose, shortness of breath), but who did not received carbocysteine. In our study, the first group is identified as group A (with carbocysteine), while the second group is identified as group B (without carbocysteine). Regarding expectorant and mucolytic medication, children in group B received drugs particular based on extracts from plants (Poligala senega and P. siberica, Uragoga ipecacuanha, Althaea officinalis), salts (sodium bicarbonate, sodium<smiles>CC(=O)NC(CS)C(=O)O</smiles>

Acetylcysteine

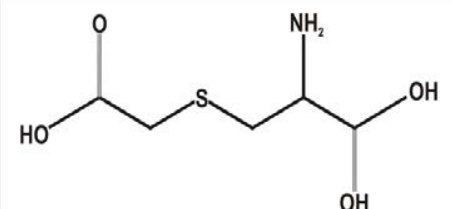

Carbocisteine
Figure 1: Acetylcysteine and carbocysteine chemical structures.

*Corresponding author: Ioan Magyar, Pharmacology Department Faculty of Medicine \& Pharmacy, University of Oradea, Romania, E-mail magyar_nelu@yahoo.com

Received September 19, 2013; Accepted November 08, 2013; Published November 15, 2013

Citation: Magyar I, Botea M, Maghiar A, Pantis C, Sava C, et al. (2013) The Rational Use of Mucolytic Drugs in Children: Worsening of the Cough as Adverse Drug Reaction after Carbocysteine in Children. Clin Exp Pharmacol 3: 140 doi:10.4172/2161-1459.1000140

Copyright: $\odot 2013$ Magyar I, et al. This is an open-access article distributed under the terms of the Creative Commons Attribution License, which permits unrestricted use, distribution, and reproduction in any medium, provided the original author and source are credited. 
benzoate, sodium iodide, potassium iodide, ammonium chloride), etheric oils (oleum therebentini, oleum thymi, eucalyptus, gomenol, menthol) and guaifensin. These substances are commonly used in various mixtures as orally solutions (including syrups).

For the group A, the data were collected using a special form recording the adverse effects, as provided by the European Drug Agency. These forms were then sent to the Pharmacovigilance Department, which belongs to the Romanian Drug Agency. Data from all children belonging to the both groups A and B were statistically analyzed using chi-square test (Figure 2).

\section{Results}

From the analysis of Figure 3, it can be seen that in the group A prevails the female sex $(57.3 \%)$, while in group B the majority is made by males $(53.4 \%)(\mathrm{p}=0.032)$.

In group A more than $68.54 \%$ patients were younger than 4 years old, comparatively with group $\mathrm{B}$, where this percentage was of $55.34 \%(\mathrm{p}=0.035)$. It is important to note that in this age group it is rarely necessary to provide mucolytics such as acetylcysteine or carbocysteine. This is because these children rarely present with chesty cough which is thick and adherent to the wall of the airways. Most often these secretions can be removed more easily with less aggressive expectorants such as those mentioned above, in group B. It is also to note that 14 children in group A are infant's category (0-1 years). We believe that the use of carbocysteine is completely unjustified in these children (Figure 4).



iil. CONCOMITANT DAUGISI AND HISTCAY

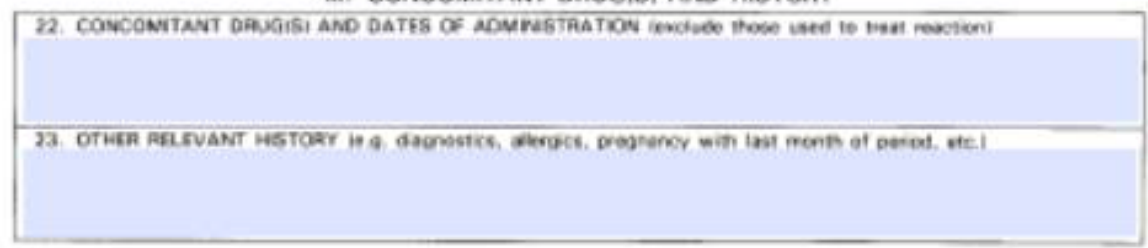

N. MANUFACTURER INFORMATION

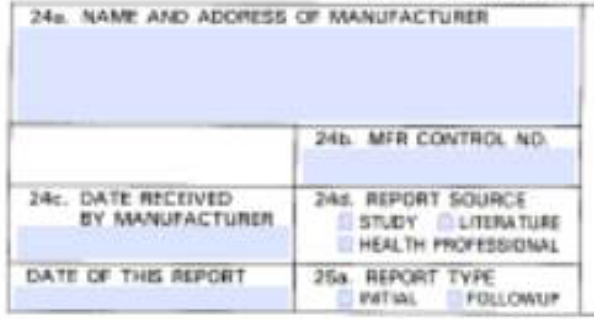

Figure 2: Pharmacovigilance department form. 


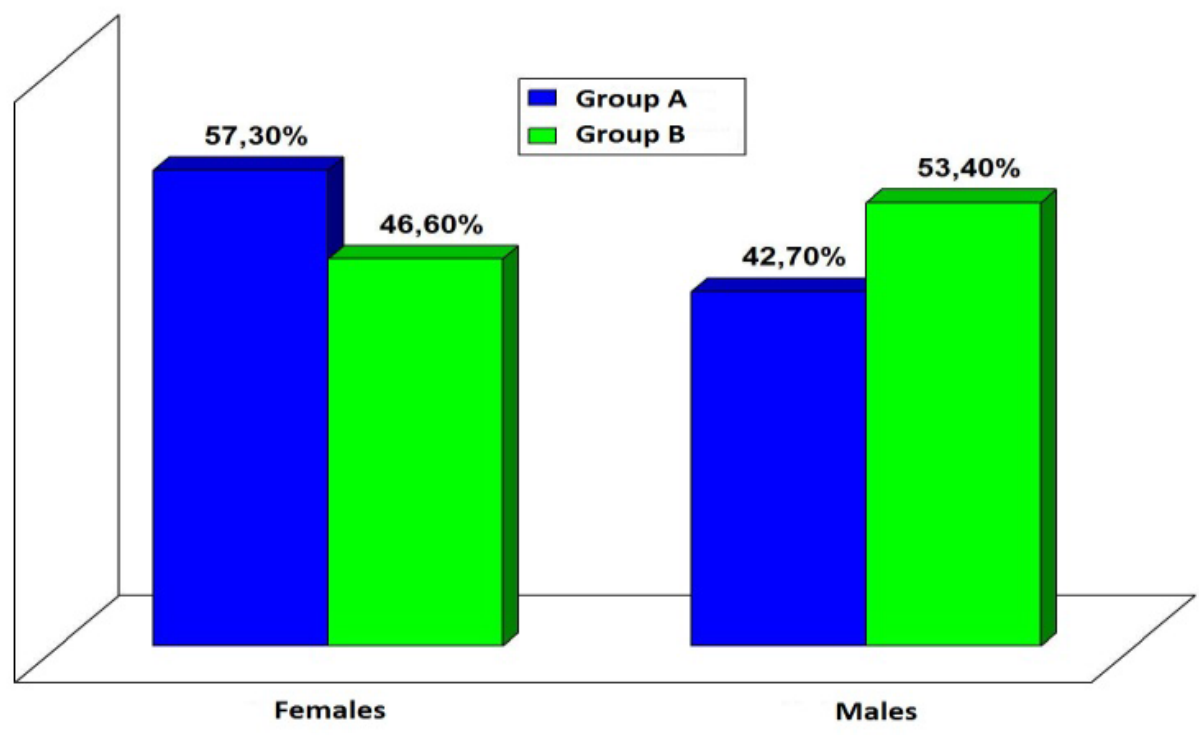

Figure 3: Cases distribution regarding sex.

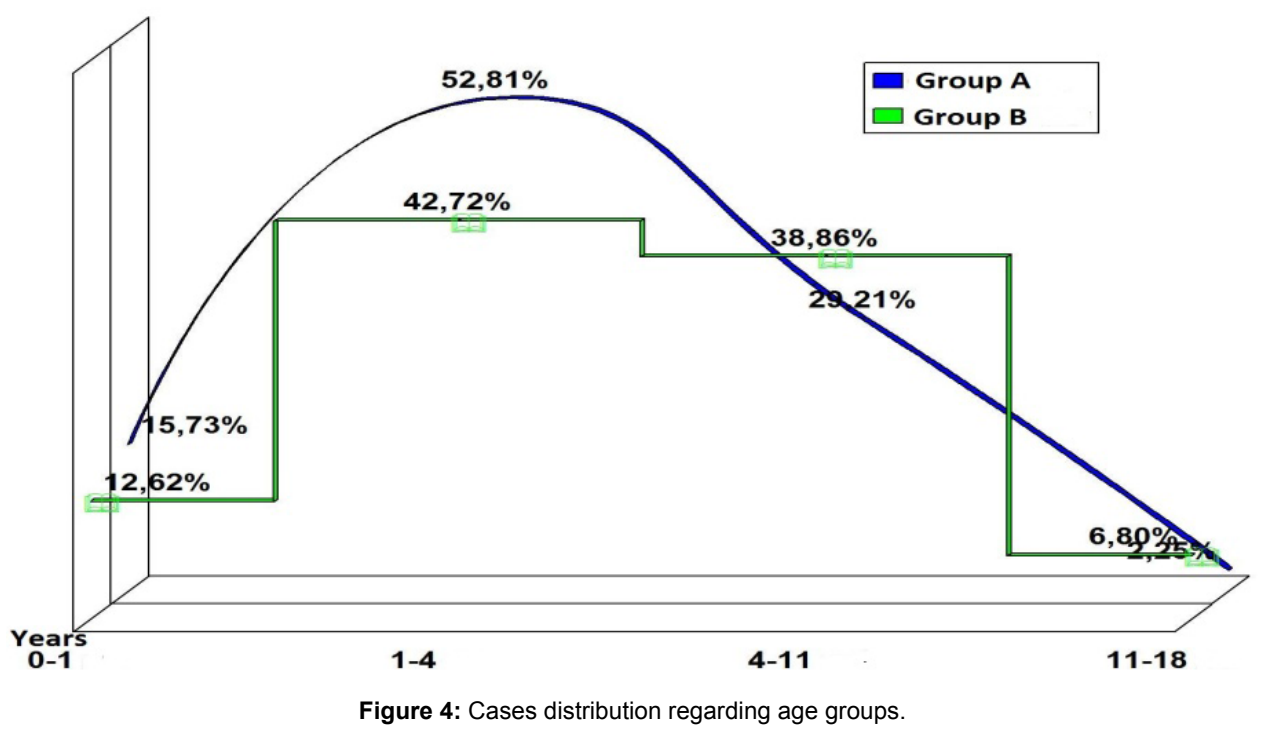

From the analysis of Figure 5 we may make the following considerations: the most frequent symptom was coughing in both groups $(93.26 \%$ and $88.35 \%$ respectively), followed by fever $(65.17 \%$ and $65.05 \%$ respectively) and rhinorrhea $(22.47 \%$ and $20.39 \%$ respectively); the most frequent association of symptoms was cough + fever + rhinorrhea $(22.47 \%$ in group A and $20.39 \%$ in group B). In both groups the main symptom was the productive cough $(46.99 \%$ and $45.05 \%$ respectively).

In group $\mathrm{A}$ it was observed that nearly $18 \%(\mathrm{n}=16)$ of the children presented vomiting associated with the use of carbocysteine.

Moreover, prolonged coughing in children can easily trigger vomiting reflex, leading them to other unpleasant consequences such as inability to ensure oral treatment. In group B, the percentage who experienced vomiting was only $9.71 \%(n=10)$.

Figure 6 shows the main types of cough presented in the two groups of patients. Analyzing the results, it is clear that there are no significant differences between the two groups of patients.

In the last figure it is shown the number of admissions of children in the two groups by age. Even if there were no of major differences noted, several observations can be drawn. In both groups the admission rate is decreasing with increasing age. Regardless of the age, the admission percentage was meaningful higher in group $B$ than in group A $(p<0.001)$. In group B we can see the percentage of the admitted patients older than 11 years $(42.86 \%)$. What can be observed is the high percentage of infants (0-1 years) and those in age category of 1-4 years, belonging to group A, requiring hospital admission. The admission to the hospital was dictated not because of prolonged cough but due to the inability to provide orally treatment (Figure 7).

\section{Conclusion}

In this study we aimed to show if there is a direct correlation 


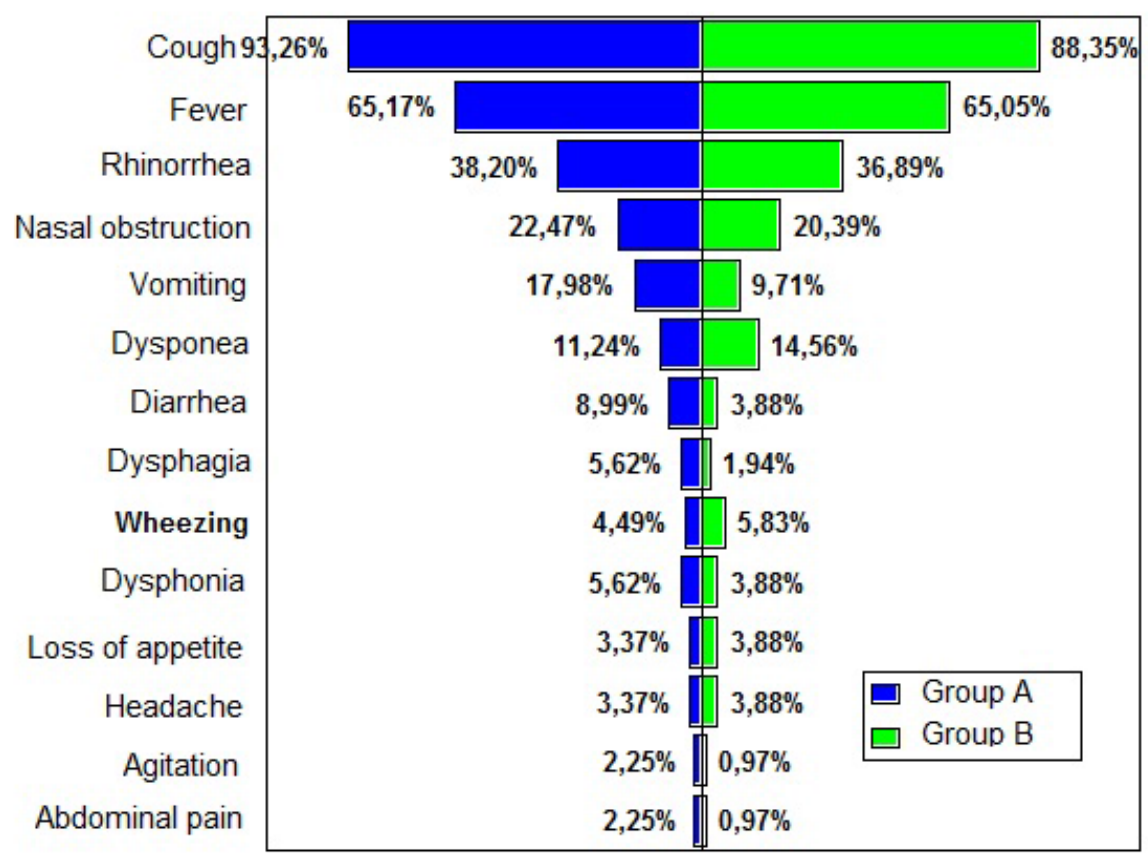

Figure 5: Cases distribution regarding associated symptoms.

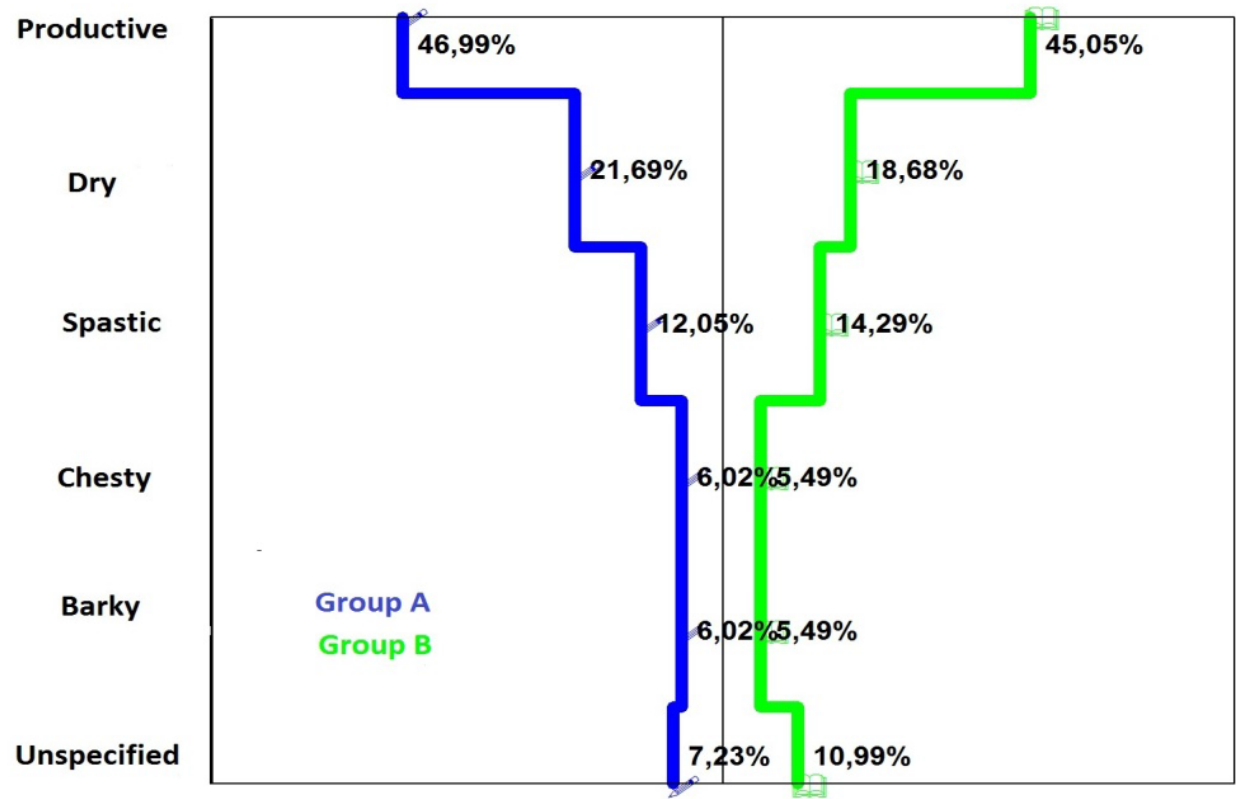

Figure 6: Cases distribution regarding cough types.

between the occurrence of cough, worsening of it, and occurrence of bronchospasm or even respiratory failure correlated with carbocysteine use.

Our research does not show the presence of respiratory failure or other life threatening symptoms that could have a direct connection with the use of carbocysteine. But we can emphasize that this drug has an irritating effect on the airways, thus, it should be used with caution in children. In all cases of worsening cough, this effect was credited anamnestic) on the use of carbocysteine.
Also, the use for a few days of carbocysteine was associated with the development of wheezing and bronchospasm.

On the other hand, worsening of cough (as frequency and duration) often causes the appearance of reflex vomiting. In children, this condition requires hospital admission and assurance of compulsory treatment with intravenous drugs. This involves discomfort for the child and family, risk of hospital infection and, ultimately, additional costs for the health care system. 


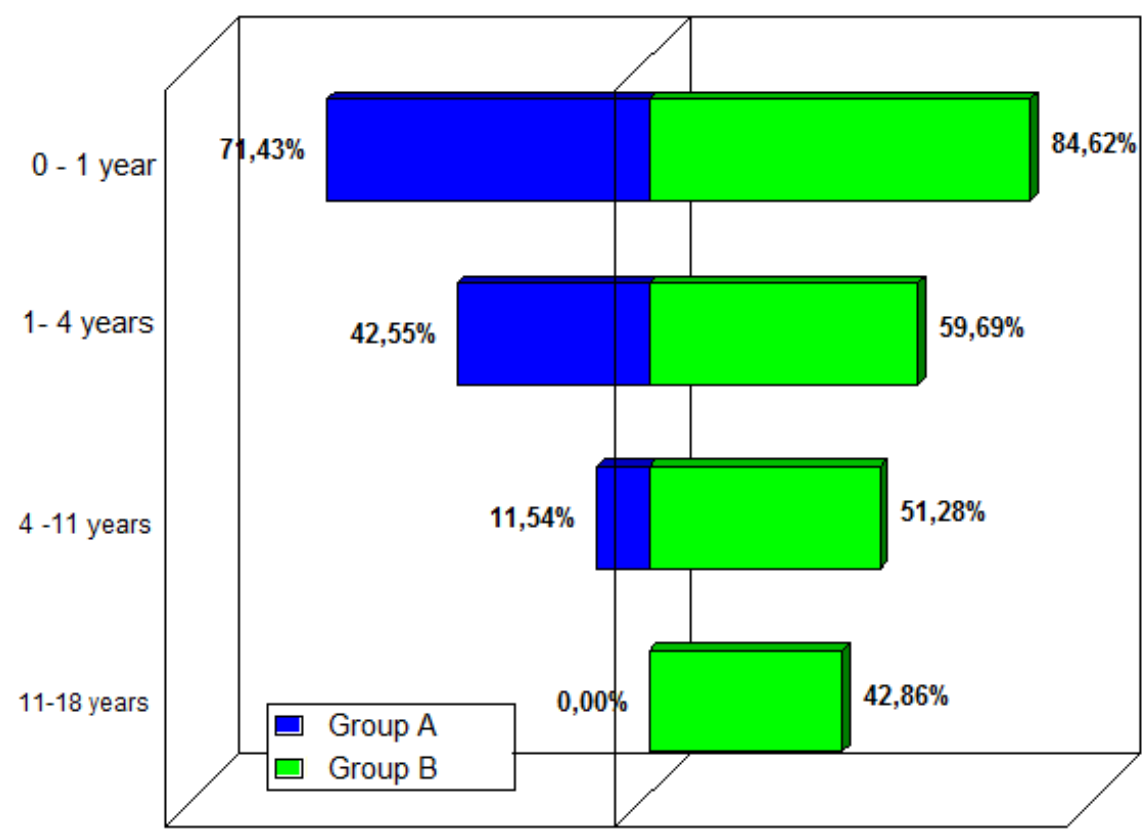

Figure 7: Hospital admission distribution by age.

In cases of asthma, recurrent wheezing or other bronchospastic diseases the use of carbocysteine should be avoided.

Generally, in the cases of children and especially in infants is advisable to provide mucolytic and expectorant drugs such as mixture of plants extract, salts or etheric (volatile) oils as those mentioned above.

We consider that there is an excessive and not justified use of carbocysteine in children. Most respiratory disease (characterized by the presence of secretions in the airways) at this age do not require mucolytic as first line treatment. Instead of, simple expectorants are more efficient.
Overall we believe that the use of carbocysteine in children exceeds the therapeutic benefit.

\section{References}

1. Katzung BG (2007) Basic and clinical pharmacology, (10thedn.) 964-965.

2. Cuparencu B (2003) Textbook of fundamental and clinical pharmacology Groupromo Edition, 2: 719-722.

3. Drug Facts and Comparisons (1997) Wolters Kluwer Company, 1090-1094.

4. Oski's Pediatrics Principles \& Practice (2006) 1387-1411.

5. Magyar I (2011) The bronchospasm and recurrent cough after carbocysteine in children. Ther Pharm and Clin Toxicology 15: 79. 